\title{
Avaliação da capacidade funcional do paciente oncogeriátrico hospitalizado
}

\author{
Functional capacity evaluation of the hospitalized oncogeriatric patient \\ Evaluación de la capacidad funcional del paciente oncogeriátrico hospitalizado \\ Esdras Edgar Batista Pereira \\ Programa de Pós-graduação em Oncologia e Ciências Médicas, \\ Universidade Federal do Pará, Belém, Pará, Brasil \\ Edilene do Socorro Nascimento Falcão Sarges \\ Serviço de Fisioterapia, Hospital Universitário João de Barros Barreto, \\ Universidade Federal do Pará, Belém, Pará, Brasil
}

Nadia Barreto dos Santos

Programa de Residência Multiprofissional em Saúde da Família,

Universidade do Estado do Pará, Belém, Pará, Brasil

\section{RESUMO}

No Brasil, estima-se que $60 \%$ dos pacientes oncológicos tenham mais de 65 anos de idade, o que obriga uma ação integrada entre a oncologia e a geriatria. Essa nova abordagem evidencia a capacidade funcional como um preditor de saúde. $O$ presente estudo tem como objetivo avaliar a capacidade funcional e o desempenho dos sistemas funcionais de idosos com diagnóstico de câncer. Trata-se de um estudo descritivo e analítico transversal, com idosos, que apresentam diagnóstico de câncer, internados na clínica médica do Hospital Universitário João de Barros Barreto, em Belém, Estado do Pará, Brasil. Os instrumentos utilizados foram: a escala Performance Status do Eastern Cooperative Oncology Group (PS-ECOG), a escala de Katz (ABVD), escala de Lawton (AIVD), miniexame do estado mental (MEEM), escala de depressão geriátrica 15 (GDS-15), teste Timed Up and Go (TUG), teste de Tinetti e a avaliação funcional das habilidades de comunicação da Associação Americana de Fonoaudiologia (ASHA FACS). Foram avaliados sete idosos (amostra por conveniência), com a capacidade funcional classificada entre 2 e 4 na escala PS-ECOG, e desempenho médio de 10,4 pontos para as ABVD e de 17,6 para as AIVD. O perfil dos sistemas funcionais evidenciou desempenho médio de 14,9 pontos no MEEM; 3,3 na escala GDS-15; 5,5 na ASHA FACS; 12,2 segundos no TUG e de 18,5 pontos no teste de Tinetti, revelando alterações na autonomia e na independência. A maioria dos indivíduos estudados apresentou sua capacidade funcional alterada, devido à semidependência para as atividades de vida diária, justificada por desempenhos anormais na cognição, mobilidade e comunicação.

Palavras-chave: Idoso Fragilizado; Hospitalização; Câncer; Geriatria; Oncologia.

\section{INTRODUÇÃO}

Nas últimas décadas, cresce o número de idosos na população mundial. Esse aumento acelerado é considerado uma conquista da humanidade, consequência das melhorias nas áreas da saúde, educação e economia, fatores que contribuem para uma maior longevidade. Mas, com a longevidade, aumentam as chances para 0 desenvolvimento de doenças, entre elas o câncer ${ }^{1,2}$.

Segundo estudos da Organização Mundial da Saúde, a mortalidade por câncer no mundo aumentará em $45 \%$, entre 2007 e 2030, em parte devido ao crescimento e

\footnotetext{
Correspondência / Correspondence / Correspondencia:

Esdras Edgar Batista Pereira

Conjunto Cidade Nova 8, WE 43B, 742. Bairro: Coqueiro

CEP: 67133-260 Ananindeua-Pará-Brasil

Tel.: +55 (91) 98300-4629
}

ao envelhecimento da população ${ }^{3}$. No Brasil, estima-se que $60 \%$ dos pacientes oncológicos tenham 65 anos de idade ou mais e que $70 \%$ das mortes decorrentes da doença ocorram na terceira idade ${ }^{4}$.

Essas informações reforçam a necessidade da ação integrada entre duas áreas da saúde, a oncologia e a geriatria, abordagem denominada de oncologia geriátrica ou oncogeriatria, capaz de compreender e tratar o idoso oncológico, levando em consideração suas especificidades ${ }^{4}$. Essa nova abordagem evidencia a capacidade funcional ou a funcionalidade como um preditor de saúde, capaz avaliar, de forma objetiva e padronizada, a capacidade física. Sua identificação pode auxiliar a predizer a tolerância ao tratamento oncológico, assim como padronizar condutas direcionadas a essa faixa etária ${ }^{5}$.

A capacidade funcional é definida como a capacidade de gerir a própria vida ou cuidar de si mesmo, e é influenciada pelo grau de autonomia e 
independência do indivíduo. Seus critérios englobam o funcionamento integrado e harmonioso das atividades diárias de vida, como a cognição, o humor, a mobilidade e a comunicação, o que permite dizer das melhores ou piores condições do idoso para enfrentar as doenças, facilitando e direcionando o planejamento do cuidado e o acompanhamento a longo prazo ${ }^{6,7}$.

objetivo deste estudo foi avaliar a capacidade funcional e o desempenho dos sistemas funcionais de idosos com diagnóstico de câncer submetidos à internação hospitalar.

\section{MATERIAIS E MÉTODOS}

Foi realizado um estudo descritivo e analítico transversal, com sete idosos (amostra por conveniência) com diagnóstico de câncer, internados na clínica médica do Hospital Universitário João de Barros Barreto (HUJBB), em Belém, Estado do Pará, Brasil, no período de março a julho de 2012. O estudo foi aprovado pelo Comitê de Ética em Pesquisa do HUJBB com protocolo $n^{\circ} 1007 / 11$, de acordo com a Resolução 196/96 do Conselho Nacional de Saúde.

\section{INSTRUMENTOS AVALIATIVOS}

Os idosos foram identificados pela sigla "P" acompanhada de um numeral e submetidos à avaliação multidimensional, que incluiu a análise dos dados demográficos (sexo, idade, estado civil, naturalidade e tempo de estudo), clínico-epidemiológicos (tipo de câncer e outras doenças diagnosticadas), do desempenho para capacidade funcional e dos principais sistemas funcionais.

\section{Avaliação da capacidade funcional}

A capacidade funcional foi avaliada por meio da escala Performance Status do Eastern Cooperative Oncology Group (PS-ECOG) e as atividades básicas de vida diárias, utilizando a escala de Katz (ABVD) e a escala de Lawton e Brody (AIVD).

A escala PS-ECOG, elaborada por Oken et al ${ }^{8}$ junto ao Eastern Cooperative Oncology Group, avalia como a doença afeta as habilidades de vida diária do paciente, com escore que varia de zero a cinco pontos, permitindo classificar o paciente com o índice 0 (totalmente ativo, capaz de continuar todo o desempenho de pré-doença, sem restrição), 1 (restritos para atividade física extenuante, porém capazes de realizar um trabalho de natureza leve ou sedentária), 2 (completamente capaz para o autocuidado, mas incapaz de realizar quaisquer atividades de trabalho; fora do leito por mais de 50\% do tempo), 3 (capacidade de autocuidado limitada, restrito ao leito ou à cadeira mais de 50\% do tempo de vigília), 4 (completamente limitado, não pode exercer qualquer autocuidado; restrito ao leito ou à cadeira) e 5 (morto) $)^{8,9}$.

A escala ABVD, construída por Katz et al ${ }^{10}$, refere-se às tarefas necessárias para o cuidado com o corpo ou autopreservação, e é composta por seis domínios, com escore total que varia de 6 a 18 pontos, permitindo classificar o idoso como independente (6 pontos), semi-dependente (7 a 16 pontos) e dependente (acima de 16 pontos) $)^{6,11}$.

A escala AIVD, desenvolvida por Lawton e Brody ${ }^{12}$, refere-se às tarefas necessárias para o cuidado com - domicílio ou atividades domésticas. É composta por nove domínios, com um escore total que varia de 9 a 27 pontos, permitindo classificar o idoso como dependente (9 pontos), semidependente $(10$ a 18 pontos) e independente (19 a 27 pontos $)^{6,13}$.

\section{Avaliação cognitiva}

A função cognitiva foi avaliada por meio do miniexame de estado mental (MEEM), elaborada por Folstein et $\mathrm{al}^{14}$, composto por 11 domínios, com o escore total que varia de 0 a 30 pontos. É um teste de rastreio, que possibilita classificar o idoso como normal ou com possível demência, considerando sua escolaridade, com escore de normalidade de 14 pontos para analfabetos, 18 pontos para aqueles que tiveram entre um e oito anos de estudo e 24 pontos para aqueles com mais de oito anos de estudo ${ }^{15}$.

\section{Avaliação do humor}

humor foi avaliado pela escala de depressão geriátrica 15 (GDS-15), construída por Yesavage et $a^{16}$, composta por 15 perguntas, com escore total que varia de 0 a 15 pontos. Por meio dessa escala pode-se obter o resultado dentro da normalidade (0 a 4 pontos), com depressão (5 a 10 pontos) ou com depressão grave (1 1 a 15 pontos) $)^{17}$.

\section{Avaliação da mobilidade}

A mobilidade foi analisada por meio do teste Time Up and Go (TUG) e do teste de Tinetti (TT). O TUG, desenvolvido por Podsiadlo e Richardson ${ }^{18}$, começa com o idoso sentado em uma cadeira com assento a $45 \mathrm{~cm}$ do chão, a seguir submetido a um trajeto de $3 \mathrm{~m}$, de ida e volta, o que é devidamente cronometrado e classificado conforme o tempo de desempenho: mobilidade normal (menor que $10 \mathrm{~s}$ ); boa mobilidade (11 a 20 s); mobilidade regular (21 a 30 s) e mobilidade prejudicada (acima de $30 \mathrm{~s})^{19}$.

$\bigcirc \mathrm{TT}$, elaborado por Tienetti et $\mathrm{al}^{20}$, avalia a mobilidade levando em consideração o risco de queda. É subdivido em 16 domínios: de 1 a 9 avalia - equilíbrio e de 10 a 16 avalia a marcha. Possui um escore que varia de 0 a 28 pontos, permitindo classificar o idoso com alto risco de queda $(<19$ pontos), risco moderado de queda (20 a 24 pontos) e baixo risco de queda ( $>24$ pontos) ${ }^{21}$.

\section{Avaliação da funcionalidade da comunicação}

A funcionalidade da comunicação foi investigada utilizando a análise quantitativa da avaliação funcional das habilidades de comunicação da associação Americana de Fonoaudiologia (ASHA FACS), elaborada por Fratalli et $a^{22}$. É composta por 26 questões, adequadas a todos os idosos entrevistados, divididas em 
quatro domínios, com um escore total que varia de 1 a 7 pontos, permitindo classificar o idoso como funcional sem auxílio (7 pontos), auxílio moderado (3 a 6 pontos) e auxílio máximo (1 a 2 pontos $)^{23}$.

\section{ANÁLISE ESTATÍSTICA}

Foi realizada a análise descritiva dos dados referentes ao perfil sociodemográfico, clínico-epidemiológico, da capacidade funcional e dos sistemas funcionais, expostos em forma de tabela. Posteriormente foi aplicado o teste correlação linear de Pearson, para avaliar a associação entre as variáveis da capacidade funcional e dos sistemas funcionais. Todo o processo estatístico contou com a utilização do software Bioestat 5.0, respeitando o nível de significância de 5\% $(\mathrm{p}<0,05)$.

\section{RESULTADOS}

No período de realização do estudo a clínica médica do HUJBB recebeu 112 idosos para internação. Destes, 94 foram submetidos à avaliação da capacidade funcional, sendo que somente sete $(6,25 \%)$ possuíam diagnóstico fechado de câncer no momento da avaliação. Os dados demográficos demonstraram que a média de idade foi de 71,57 ( \pm 8,06) anos; três pacientes eram do sexo masculino e quatro do sexo feminino, com um tempo médio de estudo de 4,43 $( \pm 1,99)$ anos (Tabela 1).
No que tange ao perfil clínico-epidemiológico identificaram-se: dois casos de câncer no pâncreas, dois casos de câncer no estômago, um caso de câncer no fígado, um caso de mieloma múltiplo e um caso de câncer no esôfago, todos associados a pelo menos uma outra doença, como hipertensão arterial, diabetes mellitus e cirrose hepática (Tabela 2).

Ao avaliar a capacidade funcional, observou-se que os idosos apresentaram uma classificação entre dois e quatro na escala PS-ECOG, com um desempenho médio de 10,4 ( $\pm 3,9)$ pontos para as ABVD e de 17,6 $( \pm 6,8)$ para as AIVD (Tabela 3$)$.

A tabela 4 mostra o perfil dos sistemas funcionais investigados, descrevendo um desempenho médio de 14,9 ( $\pm 5,5)$ pontos no MEEM, de 3,3 ( $\pm 2,9)$ na escala GDS-15 e de 5,5 ( $\pm 1,4)$ na ASHA FACS. Dos sete pacientes avaliados, três não foram submetidos aos testes de mobilidade porque dois estavam restritos ao leito e um necessitava de ajuda frequente para locomoção. Os demais foram submetidos aos testes e apresentaram um desempenho médio de 12,2 ( $\pm 2,5)$ seg no TUG e 18,5 ( \pm 4,5) no TT.

A correlação entre as variáveis da capacidade funcional e os sistemas funcionais, exposta na tabela 5, revelou que as atividades de vida diária tiveram significativa associação com o desempenho dos domínios relacionados à independência.

Tabela 1 - Perfil demográfico dos idosos com câncer na clínica médica do HUJBB, em Belém, Estado do Pará, Brasil, 2012

\begin{tabular}{cccccc}
\hline Identificação & Sexo & Idade & Estado civil & Naturalidade & Escolaridade (anos) \\
\hline P1 & M & 73 & Casado & Belém, Pará & 4 \\
P2 & F & 75 & Viúvo & Japerica, Pará & 7 \\
P3 & F & 65 & Solteiro & Moju, Pará & 5 \\
P4 & M & 65 & Separado & Belém, Pará & 6 \\
P5 & F & 81 & Casado & Muaná, Pará & 5 \\
P6 & F & 61 & Casado & Fortaleza, Ceará & 3 \\
P7 & M & 81 & Casado & Bragança, Pará & 1 \\
\hline
\end{tabular}

Tabela 2 - Perfil clínico-epidemiológico dos idosos com câncer na clínica médica do HUJBB, em Belém, Estado Pará, Brasil, 2012

\begin{tabular}{ccccc}
\hline \multirow{2}{*}{ Identificação } & Tipo de câncer & \multicolumn{3}{c}{ Outras doenças diagnosticadas } \\
\cline { 3 - 5 } & Pâncreas & Hipertensão & Diabetes mellitus & Cirrose hepática \\
\hline P1 & Estômago & + & - & - \\
P2 & Pâncreas & + & + & - \\
P3 & Fígado & + & - & + \\
P4 & Mieloma múltiplo & - & + & - \\
P5 & Estômago & + & - & + \\
P6 & Esôfago & - & + & - \\
P7 & & - & +
\end{tabular}

+: Presente; -: Ausente. 
Tabela 3 - Perfil da capacidade funcional dos idosos com câncer na clínica médica do HUJBB em Belém, Estado do Pará, Brasil, 2012

\begin{tabular}{cccc}
\hline Identificação & PS-ECOG & ABVD & AIVD \\
\hline P1 & 3 & 14 & 9 \\
P2 & 4 & 13 & 16 \\
P3 & 2 & 6 & 27 \\
P4 & 2 & 6 & 19 \\
P5 & 4 & 15 & 14 \\
P6 & 2 & 7 & 26 \\
P7 & 3 & 12 & 12 \\
\hline
\end{tabular}

Tabela 4 - Perfil dos sistemas funcionais dos idosos com câncer na clínica médica do HUJBB em Belém, Estado do Pará, Brasil, 2012

\begin{tabular}{cccccc}
\hline Identificação & MEEM & GDS-15 & TUG & TT & ASHA FACS \\
\hline P1 & 9 & 9 & Não realizado & Não realizado & 3,0 \\
P2 & 19 & 4 & Não realizado & Não realizado & 5,9 \\
P3 & 23 & 2 & 12 & 25 & 6,4 \\
P4 & 13 & 3 & 15 & 16 & 6,1 \\
P5 & 18 & 1 & Não realizado & Não realizado & 6,4 \\
P6 & 14 & 0 & 9 & 18 & 6,6 \\
P7 & 8 & 4 & 13 & 15 & 3,9 \\
\hline
\end{tabular}

Tabela 5 - Matriz de coeficientes de correlação entre as variáveis da funcionalidade dos idosos com câncer na clínica médica do HUJBB em Belém, Estado do Pará, Brasil, 2012

\begin{tabular}{|c|c|c|c|c|c|c|c|}
\hline & PS-ECOG & ABVD & AIVD & MEEM & GDS-15 & TUG & TT \\
\hline ABVD & $0,910^{*}$ & & & & & & \\
\hline AIVD & $-0,660$ & $-0,841^{*}$ & & & & & \\
\hline MEEM & 0,063 & $-0,243$ & 0,612 & & & & \\
\hline GDS-15 & 0,207 & 0,448 & $-0,715$ & $-0,518$ & & & \\
\hline TUG & $0,842^{*}$ & $0,837^{*}$ & $-0,686$ & 0,051 & 0,494 & & \\
\hline $\mathrm{TT}$ & $-0,863^{*}$ & $-0,891^{*}$ & $0,764^{*}$ & 0,163 & $-0,466$ & $-0,952^{*}$ & \\
\hline ASHA FACS & $-0,164$ & $-0,488$ & $0,761^{*}$ & 0,754 & $-0,887^{*}$ & $-0,274$ & 0,333 \\
\hline
\end{tabular}

* p-valor $<0,05$.

\section{DISCUSSÃO}

A avaliação da capacidade funcional do idoso oncológico pode permitir a compreensão de fatores que vão além da idade cronológica, tais como expectativa e qualidade de vida, riscos e benefícios de tratamentos e pode auxiliar no desenvolvimento de intervenções para grupos de idosos mais frágeis. A identificação das variáveis dessa avaliação pode ajudar a prevenir os impactos do tratamento do câncer sobre o estado funcional ${ }^{24}$.

Dos pacientes incluídos neste estudo encontraram-se quatro que apresentaram um índice de três ou quatro na escala do PS-ECOG, mostrando que a maioria dos avaliados possuía algum comprometimento que limitava suas habilidades diárias. Segundo Kristjansson et $\mathrm{a}^{25}$, somente a avaliação do PS-ECOG não informa completamente a gama de problemas que o paciente idoso oncológico pode ter, havendo necessidade de se investigar outros domínios da avaliação geriátrica ampla, como a presença da dependência funcional, das comorbidades, da polifarmácia, da desnutrição, da disfunção cognitiva e da depressão. E, ainda, questões relacionadas ao suporte social e às síndromes geriátricas.

Por isso, para complementar o PS-ECOG, foi identificado o nível de dependência funcional para as atividades de vida diária, quando se observou um desempenho médio de 10,4 pontos para as ABVD e de 17,6 para as AIVD, permitindo dizer que, em média, os idosos eram semidependentes para ambas as atividades. A presença de algum grau de comprometimento funcional na maioria dos idosos avaliados difere dos achados de outros estudos, como o de Wildes et $\mathrm{al}^{26}$, que, ao avaliarem 65 idosos de um hospital universitário de uma cidade de Missouri, nos Estados Unidos, identificaram que somente 10,8\% eram dependentes para as $A B V D$ e $38,5 \%$ eram dependentes para as AIVD. 
Esses resultados podem ainda ser variáveis conforme o nível da assistência à saúde, seja ela ambulatorial ou hospitalar. Por exemplo, os achados do presente estudo são contrários, também, aos encontrados por Lera et $\mathrm{al}^{27}$, que, ao estudarem 40 idosos com câncer de um serviço ambulatorial de oncologia clínica de São Paulo, classificaram sua amostra como independente para as atividades de vida diária.

Identificar as variáveis relacionadas ao perfil da capacidade funcional do idoso com câncer pode ajudar na determinação do prognóstico clínico e funcional após o tratamento ${ }^{28}$. Fukata et a ${ }^{29}$ afirmam, em seu estudo, que a avaliação atividades da vida diária é muito importante para mensurar o sucesso do tratamento cirúrgico em idosos, visto que, para alguns, por vezes esse tratamento é considerado inadequado, especialmente para aqueles com comorbidades. Por isso, além da identificação do desempenho para as atividades de vida diária, o conhecimento sobre o nível de autonomia e independência deve ser avaliado para melhor compreensão dos sistemas funcionais envolvidos.

Dos idosos investigados, apenas quatro indivíduos apresentaram um escore que os classificou com quadro de demência ou comprometimento cognitivo no MEEM, e apenas um apresentou pontuação que indica quadro depressivo na GDS-15. Esses dados corroboram com os de Shin et $\mathrm{a}^{30}$, que estudaram 64 idosos de um hospital universitário de Seul e observaram que a maioria apresentou déficit cognitivo $(56,3 \%)$ e humor preservado $(59,4 \%)$. Já os achados de Lazarovici et a ${ }^{31}$ que avaliaram 65 idosos de um hospital de Paris, revelaram uma incidência de $45,3 \%$ de déficit cognitivo e de $47,6 \%$ de quadro depressivo.

A investigação dos domínios relacionados à independência revelou que três idosos não realizaram os testes da mobilidade devido a limitações para locomoção. Os que executaram, apresentaram uma mobilidade normal ou boa no TUG, mas três desses obtiveram uma pontuação que revelou alto risco de queda no teste $T T$, evidenciando comprometimento da marcha e do equilíbrio. No domínio comunicação, os idosos obtiveram uma pontuação 5,5 pontos no ASHA FACS, revelando a necessidade de um auxílio moderado para realização dessa tarefa. Isso mostra que a maioria dos idosos inclusos na pesquisa apresentou sua independência alterada.

Os dados referentes à mobilidade foram similares aos encontrados por Overcahs e Rivera ${ }^{32}$, que aplicaram - TUG em 68 idosos com câncer e identificaram um desempenho médio de 14,2 s. Bellera et $\mathrm{a}^{33} \mathrm{e}$ Soubeyran et $\mathrm{a}^{34}$ investigaram a mobilidade de outra população e observaram que cerca de $25 \%$ desses apresentaram anormalidades no desempenho do TUG. No estudo de Ferste ${ }^{35}$, o TT foi aplicado em 90 idosos internados em um hospital de Curitiba, e revelou um desempenho de 22,1 pontos, indicando um risco moderado de queda. Assim como Gosh et a ${ }^{36}$, que avaliaram a mobilidade de 258 pacientes de um hospital austríaco, e observaram um desempenho entre 21,3 e 53,7 s no TUG e entre 15,9 e 19,2 pontos no TT.

Conhecer as características da mobilidade (velocidade, equilíbrio e qualidade da marcha) do idoso oncológico facilita a elaboração de estratégias preventivas que possam minimizar a ocorrência de comorbidades que comprometem 0 tratamento oncológico, tais como as quedas, e, consequentemente, a fratura, as limitações e a imobilidade. A utilização do TUG e do TT são complementares, pois revelam particularidades distintas que necessitam ser investigadas ${ }^{37}$.

Neste estudo reforça-se a presença de diversos fatores associados com a capacidade funcional de idosos oncológicos hospitalizados. A autonomia e a independência são as variáveis determinantes para - bom desempenho das atividades de vida diária, podendo ter associação direta com os sistemas funcionais. A utilização dos instrumentos avaliativos, abordados no presente estudo, pode contribuir significativamente na elaboração de planos de cuidados para assistência em oncogeriatria.

O estudo realizado apresentou limitações importantes quanto à amostra. Por se tratar de um estudo piloto, a amostra é pequena, heterogênea e não representativa para a elaboração de maiores conclusões. Os resultados encontrados devem ser considerados apenas para a população do estudo, cabendo comparações com outros grupos apenas a título de ilustração sobre a importância da avaliação da capacidade funcional em oncogeriatria.

\section{CONCLUSÃO}

A maior parte da população estudada apresentou sua capacidade funcional alterada, devido à semidependência para as atividades de vida diária, justificada por desempenhos anormais na autonomia e independência. Os sistemas funcionais mais comprometidos foram a cognição, os domínios relacionados à mobilidade e à comunicação.

Estratégias similares ao estudo mostram-se importantes no contexto clínico da oncogeriatria, pois a avaliação da capacidade funcional pode contribuir significativamente na elaboração de planos de cuidados para assistência ao idoso oncológico. Por meio da identificação da funcionalidade norteiam-se medidas que previnem ou reduzem o comprometimento funcional, diminuindo os riscos de complicações e melhorando a qualidade de vida do idoso com câncer.

\section{AGRADECIMENTOS}

À prof. ${ }^{a}$ dr. ${ }^{a}$ lêda Maria Louzada Guedes, Programa de Atenção Integral ao Idoso da Amazônia Brasileira, Ministério da Educação, Universidade Federal do Pará, Núcleo de Pesquisa em Oncologia do HUJBB e a Coordenação de Aperfeiçoamento de Pessoal de Nível Superior. 


\title{
Functional capacity evaluation of the hospitalized oncogeriatric patient
}

\begin{abstract}
In Brazil, it is estimated that $60 \%$ of cancer patients are over 65 years old which requires an integrated action between oncology and geriatrics. This new approach shows the functional capacity as a predictor of health. The present study aims to evaluate the functional capacity and the performance of the functional systems of older adults diagnosed with cancer. This is a descriptive and analytical cross-sectional study with the elderly people, who have diagnosis of cancer, admitted to the medical clinic of Hospital Universitário João de Barros Barreto in Belém, Pará State, Brazil. The instruments used were: Eastern Cooperative Oncology Group the Performance Status scale (ECOG-PS), the Katz scale (ABVD), the Lawton scale (IADL), Mini Mental State Examination (MMSE), Geriatric Depression Scale 15 (GDS-15), Timed Up and Go test (TUG), Tinetti test and the functional assessment of communication skills of the American Association of Speech-Hearing Association (ASHA FACS). Seven elderly people (convenience sample) were evaluated with their functional capacity classified between 2 and 4 in ECOG-PS scale and their average performance of 10.4 points for ABVD and 17.6 for IADL. The profile of functional systems showed average performance of 14.9 points on the MMSE; 3.3 on the GDS-15 scale; 5.5 in ASHA FACS; 12.2 seconds for the TUG and 18.5 points in the Tinetti test, revealing changes in autonomy and independence. Most of those elderly patients had changed their functional capacity because of their semi-dependence for daily activities, justified by abnormal performance in cognition, mobility and communication.
\end{abstract}

Keywords: Frail Elderly; Hospitalization; Cancer; Geriatrics; Medical Oncology.

\section{Evaluación de la capacidad funcional del paciente oncogeriátrico hospitalizado}

\section{RESUMEN}

En Brasil, se estima que 60\% de los pacientes oncológicos tienen más de 65 años de edad, lo que impone una acción integrada entre la oncología y la geriatría. Ese nuevo enfoque evidencia la capacidad funcional como un predictor de salud. El presente estudio tiene como objetivo evaluar la capacidad funcional y el desempeño de los sistemas funcionales de adultos mayores con diagnóstico de cáncer. Se trata de un estudio descriptivo y analítico transversal, con adultos mayores que presentan diagnóstico de cáncer, internados en la clínica médica del Hospital Universitário João de Barros Barreto, en Belém, Estado de Pará, Brasil. Los instrumentos utilizados fueron: la escala Performance Status del Eastern Cooperative Oncology Group (PS-ECOG), la escala de Katz (ABVD), escala de Lawton (AIVD), mini examen del estado mental (MEEM), escala de depresión geriátrica 15 (GDS-15), test Timed Up and Go (TUG), test de Tinetti y la evaluación funcional de las habilidades de comunicación de la Asociación Americana de Habla, Lenguaje y Audición (ASHA FACS). Fueron evaluados siete adultos mayores (muestra por conveniencia), con capacidad funcional clasificada entre 2 y 4 en la escala PS-ECOG, y desempeño medio de 10,4 puntos para las ABVD y de 17,6 para las AIVD. El perfil de los sistemas funcionales evidenció un desempeño medio de 14,9 puntos en el MEEM; 3,3 en la escala GDS-15; 5,5 en la ASHA FACS; 12,2 segundos en el TUG y de 18,5 puntos en el test de Tinetti, revelando alteraciones en la autonomía y en la independencia. La mayoría de los individuos estudiados presentó su capacidad funcional alterada, debido a la semidependencia para las actividades de la vida diaria, justificada por desempeños anormales en la cognición, movilidad y comunicación.

Palabras clave: Anciano Frágil; Hospitalización; Cáncer; Geriatría; Oncología Médica.

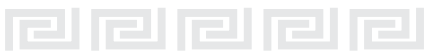

\section{REFERÊNCIAS}

1 Soares LC, Santana MG, Muniz RM. O fenômeno do câncer na vida de idosos. Cienc Cuid Saude. 2010 out-dez;9(4):660-7.

2 Visentin A, Lenardt $\mathrm{MH}$. $\bigcirc$ itinerário terapêutico: história oral de idosos com câncer. Acta Paul Enferm. 2010;23(4):486-92.

3 Pirajá FCS, Lages RB, Costa UA, Teles JBM, Campelo V. Sobrevida de pacientes com câncer de próstata. Rev Bras Promo Saude. 2013 janmar;26(1):45-50.
4 Assis CMRB, Melo HMA, Melo EMA, Kitner D, Costa Júnior Jl. Oncologia geriátrica: conceitos, tendências e desafios. Geriatr Gerontol. $2011 ; 5(2)$ : 106-11.

5 Cerullo F, Colloca G, Ferrini A, Ciaburri M, Scambia $G$, Bernabei $R$, et al. Misure di funzione fisica in oncogeriatria. G Gerontol. 2011 ;5(9):265-72.

6 Moraes EN. Atenção à saúde do idoso: aspectos conceituais. Brasília: Organização Pan-Americana da Saúde; 2012. 98 p. 
7 Karnakis T. Oncogeriatria: uma revisão da avaliação geriátrica ampla nos pacientes com câncer. RBM. 2011 mai;68(2):8-12.

8 Oken MM, Creech RH, Tormey DC, Horton J, Davis TE, McFadden ET, et al. Toxicity and response criteria of the Eastern cooperative oncology group. Am J Clin Oncol. 1982 Dec;5(6):649-55.

9 Machado L, Saad IAB, Honma HN, Morcillo AM, Zambon L. Evolução do status de performance, índice de massa corpórea e distância percorrida no teste de caminhada de seis minutos em pacientes com câncer de pulmão avançado submetidos à quimioterapia. J Bras Pneumol. 2010 setout;36(5):588-94.

10 Katz S, Ford AB, Moskowitz RW, Jackson BA, Jaffe MW. Studies of illness in the aged. The index of ADL: a standardized measure of biological and psychosocial function. JAMA. 1963 Sep;185(12): 914-9.

11 Afonso MS, Silveira KS, Soares MP, Lopes JF, Azevedo P, Brito LCN. Déficits funcionais de idosos correlacionados a cada década de vidas. Rev Inspirar Mov Saude. 2013 jun-jul;5(2):1-6.

12 Lawton MP, Brody EM. Assessment of older people: self maintaining and instrumental activities of daily living. Gerontologist. 1969;9(3 Part 1):179-86.

13 Prefeitura de Florianópolis. Secretaria Municipal de Saúde. Diretoria da Atenção Primária à Saúde. Protocolo de atenção à saúde do idoso. Tubarão: Copiart; 2011.

14 Folstein MF, Folstein SE, McHugh PR. Mini-mental state: a practical method for grading the cognitive state of patients for the clinician. J Psychiatr Res. 1975 Nov; 12(3): 189-98.

15 Razali R, Jean-Li L, Jaffar A, Ahmad M, Shah SA, Ibrahim N, et al. Is the Bahasa Malaysia version of the Montreal Cognitive Assessment (MoCA-BM) a better instrument than the Malay version of the Mini Mental State Examination (M-MMSE) in screening for mild cognitive impairment $(\mathrm{MCl})$ in the elderly? Compr Psychiatry. 2014 Jan;55 Suppl 1:S70-5.

16 Yesavage JA, Brink TL, Rose TL, Lum O, Huang V, Adey $M$, et al. Development and validation of a geriatric depression screening scale: a preliminary report. J Psychiat Res. 1983;17(1):37-49.

17 Ferrari JF, Dalacorte RR. Uso da escala de depressão geriátrica de Yesavage para avaliar a prevalência de depressão em idosos hospitalizados. Sci Med. 2007 jan-mar; 17(1):3-8.

18 Podsiadlo D, Richardson S. The timed "Up \& Go": a test of basic functional mobility for frail elderly persons. J Am Geriatr Soc. 1991 Feb;39(2):142-8.

19 Martins FP, Maia HU, Pereira LSM. Desempenho de idosos em testes funcionais e o uso de medicamentos. Fisioter Mov. 2007 jan-mar;20(1):85-92.
20 Tinetti ME, Baker DI, McAvay G, Claus EB, Garrett $P$, Gottschalk $M$, et al. A multifactorial intervention to reduce the risk of falling among elderly people living in the community. $N$ Engl J Med. 1994 Sep;331 (13):821-7.

21 Silva A, Almeida JMG, Cassilhas CR, Cohen M, Peccin MS, Tufik $S$, et al. Equilíbrio, coordenação e agilidade de idosos submetidos à prática de exercícios físicos resistidos. Rev Bras Med Esporte. 2008 mar-abr; 14(2):88-93.

22 Frattali CM, Thompson CM, Holland $\mathrm{AL}$, Wohl CB, Ferketic MM. The FACS of life ASHA facs a functional outcome measure for adults. ASHA. 1995 Apr;37(4):40-6.

23 Carvalho IAM. Avaliação funcional das habilidades da comunicação: ASHA FACS para população com doença de Alzheimer [tese]. São Paulo (SP): Universidade de São Paulo, Faculdade de Medicina; 2006.

24 Hurria A, Cohen HJ, Extermann M. Geriatric oncology research in the cooperative groups: a report of a SIOG special meeting. J Geriatr Oncol. 2010 Jun; $1(1): 40-4$.

25 Kristjansson SR, Jordhoy MS, Nesbakken A, Skovlund E, Bakka A, Johannessen $\mathrm{HO}$, et al. Which elements of a comprehensive geriatric assessment (CGA) predict post-operative complications and early mortality after colorectal cancer surgery? J Geriatr Oncol. 2010 Oct; 1 (2):57-65.

26 Wildes TM, Ruwe AP, Fournier C, Gao F, Carson KR, Piccirillo JF, et al. Geriatric assessment is associated with completion of chemotherapy, toxicity and survival in older adults with cancer. J Geriatr Oncol. 2013 Jul;4(3):227-34.

27 Lera AT, Miranda MC, Trevizan LLB, Antonangelo DV, Zanellato RM, Tateyama LTC, et al. Aplicação do instrumento termômetro de estresse em pacientes idosos com câncer: estudo piloto. Rev Bras Clin Med. 2011 mar-abr;9(2):112-6.

28 Audisio RA, Papa D, Ramesh HSJ, Gennari R, Leeuwen BL, Corsini $G$, et al. Shall we operate? Preoperative assessment in elderly cancer patients (PACE) can help: a SIOG surgical task force prospective study. Crit Rev Oncol Hematol. 2008 Feb;65(2):156-63.

29 Fukata S, Ando M, Amemiya T, Kuroiwa K, Oda K. Postoperative function following radical surgery in gastric and colorectal cancer patients over 80 years of age: an objection to "ageism". Nagoya J Med Sci. 2012 Aug;74(3-4):241-51.

30 Shin DY, Lee JO, Kim YJ, Park MS, Lee KW, Kim $\mathrm{Kl}$, et al. Toxicities and functional consequences of systemic chemotherapy in elderly Korean patients with cancer: a prospective cohort study using Comprehensive Geriatric Assessment. J Geriatr Oncol. 2012 Oct;3(4):359-67. 
31 Lazarovici C, Khodabakhshi R, Leignel D, FabreGuillevin E, Minarda A, Gisselbrechta M. Factors leading oncologists to refer elderly cancer patients for geriatric assessment. J Geriatr Oncol. 2011 Jul;2(3):194-9.

32 Overcash JA, Rivera Jr HR. Physical performance evaluation of older cancer patients: a preliminary study. Crit Rev Oncol Hematol. 2008 Dec;68(3):233-4 1 .

33 Bellera CA, Rainfray $M$, Mathoulin-Pélissier S, Mertens C, Delva F, Fonck M, et al. Screening older cancer patients: first evaluation of the G-8 geriatric screening tool. Ann Oncol. 2012 Jan;23(8):216672.

34 Soubeyran P, Fonck M, Blanc-Bisson C, Blanc JF, Ceccaldi J, Mertens C, et al. Predictors of early death risk in older patients treated with first-line chemotherapy for cancer. J Clin Oncol. 2012 May;30(15): 1829-34.
35 Ferste MA. Sistema conexionista baseado em avaliação geriátrica ampla para auxílio ao diagnóstico de declínio cognitivo [dissertação]. Curitiba (PR): Universidade Tecnológica Federal do Paraná, Programa de Pós-graduação em Engenharia Elétrica e Informática Industrial; 2006.

36 Gosch M, Joosten-Gstrein B, Heppner HJ, Lechleitner M. Hyponatremia in geriatric in hospital patients: effects on results of a comprehensive geriatric assessment. Gerontology. 2012 Aug;58(5): 30-40.

37 Karuka AH, Silva JAMG, Navega MT. Análise da concordância entre instrumentos de avaliação do equilíbrio corporal em idosos. Rev Bras Fisioter. 2011 nov-dez;15(6):460-6.

Recebido em / Received / Recibido en: 28/2/2014 Aceito em / Accepted / Aceito en: 12/6/2014 